\title{
Interval Analysis of Dynamic Response of Structures
}

\author{
M. Liu \\ Nanjing Nanxijiang Tung Street 58 Jiangsu Posts \& Telecommunications Planning Designing Institute Co.Ltd
}

China

\begin{abstract}
In this paper, an interval method for the dynamic response of structures with uncertain parameters is proposed. The structural physical parameters and loads are considered as interval variables. The structural stiffness matrix, mass matrix and loading vectors are described as the sum of two parts corresponding to the deterministic matrix and the uncertainty of the interval parameters. The interval problem is then transformed into an approximate deterministic problem. The Laplace transform is used to convert the equations of the dynamic system into linear algebra equations. The effectiveness of the proposed method is demonstrated by a numerical example of a three-story structure. The results show that the range between upper and lower bounds of dynamic responses due to uncertain system parameters is narrow and acceptable. Since the presented method neglects the second order terms in the expansion of functions, the application of the approach is limited to the cases where the uncertainties of the parameters are small.
\end{abstract}

\section{Keywords-interval method; dynamic}

\section{INTRODUCTION}

In engineering design, it is important to calculate response quantities such as the displacement, stress and vibration responses against a given set of design parameters. However, in most practical situations, the structural parameters are often uncertain, such as the inaccuracy of the measurement, errors in the manufacturing and assembly process, invalidity of some components and uncertainty in boundary conditions etc. The uncertainties of structural parameters may lead to large and unexpected excursion of responses that may lead to drastic reduction in accuracy and precision of the operation. Therefore, the concept of uncertainty plays an important role in the investigation of various engineering problems. A number of methods have been developed which include uncertain model properties in the finite element analysis and aim at the quantification of the uncertainty on the analysis result. For problems with distribution description of the variety in the system parameters, probability theory is the traditional approach to handle uncertainty. One of the classical probabilistic methods (Au 2004[1]) is the Monte Carlo simulation or the stochastic finite element method. However, probabilistic modeling cannot handle situations with incomplete or little information on which to evaluate a probability, or when that information is nonspecific, ambiguous, or conflicting. Therefore, in that case, the interval method is widely used. In the interval analysis method, an uncertain structural parameter can be described as an interval variable and only its lower and upper bounds are required.

Since the mid-1960s, a new method called the interval analysis has appeared. Moore [8](1966), Alefeld and Herzberger[2](1983)have done the pioneering work. Mathematically, linear interval equations and nonlinear interval equation have been resolved partly. Because of the complexity of the algorithm, it is difficult to apply these results to practical engineering problems. Recently, a few of the interval analysis methods (Chen et al 2002[3]; Qiu and Wang 2003[9], 2005[10]; Chen and Wu 2004[4]; Chen and Zhang 2006[5]) were presented to make the interval method easier to deal with the dynamic response analysis for the complex structures with interval parameters. Although plenty of effort has been made and improvement has been achieved in the interval method to solve the uncertainty problems, the overestimation problem still existed in their studies. Hence, it is necessary to develop an effective method to solve the overestimation in the interval mathematics in the structural dynamic analysis.

In this paper, a new interval analysis method will be presented. A brief review of the interval mathematics is given first. The formulation of the dynamic response analysis for structures with interval parameters is then presented. The structural stiffness matrix, mass matrix and loading vectors are described as the sum of two parts corresponding to the deterministic matrix and the uncertainty of the interval parameters. Later, the interval problem is transformed into an approximate deterministic problem. The Laplace transform is used to convert the equations of the dynamic system into linear algebra equations. Finally, a three-story structure for the cases with and without damping is studied and the dynamic responses of this structure are calculated to demonstrate the applicability of the presented method.

\section{INTERVAL ARITHMETIC}

In this paper, real quantities will be introduced in nonbold face and interval quantities (interval number, interval vector, interval matrix) will be denoted in bold face by attaching a superscript "I" (Hansen 1992)[6],

$$
\boldsymbol{x}^{I}=[\underline{x}, \bar{x}],\{x \in R: \underline{x} \leq x \leq \bar{x}\},
$$


Where $x$ and $\bar{x}$ are the lower and upper bounds of the interval number $\boldsymbol{x}^{I}$ respectively. The set of all the closed real intervals is denoted by $I(R)$.

The mid-point and uncertainty of an interval $\boldsymbol{x}^{\boldsymbol{I}}$ are defined as

$$
x^{c}=\operatorname{mid}\left(x^{I}\right)=\frac{1}{2}(\underline{x}+\bar{x})
$$

And

$$
\Delta x=\operatorname{rad}\left(x^{I}\right)=\frac{1}{2}(\bar{x}-\underline{x})
$$

Respectively. So an interval $\boldsymbol{x}^{\boldsymbol{I}}$ can be expressed as

$$
\boldsymbol{x}^{I}=\left[x^{c}-\Delta x, x^{c}+\Delta x\right]
$$

The relative uncertainty of $\boldsymbol{x}^{I}$ is defined as

$$
\delta=\frac{\Delta x^{I}}{\left|x^{c}\right|}
$$

So an interval $\boldsymbol{x}^{\boldsymbol{I}}$ can also be expressed as

$$
\boldsymbol{x}^{I}=\left[x^{c}-\delta\left|x^{c}\right|, x^{c}+\delta\left|x^{c}\right|\right]=x^{c}+x^{c} \boldsymbol{\delta}^{I}
$$

An interval vector is a vector whose components are interval numbers. An interval matrix is a matrix whose elements are interval numbers. The set of $m \times n$ interval matrices is denoted by $\boldsymbol{I} \boldsymbol{R}^{m \times n}$. An interval matrix $\boldsymbol{A}^{\boldsymbol{I}}=$ $[\underline{A}, \bar{A}]=\left(\boldsymbol{A}_{\boldsymbol{i j}}^{\boldsymbol{I}}\right)$ is interpreted as a set of real $m \times n$ matrices by the convention,

$\boldsymbol{A}^{I}=\left\{A \in R^{m \times n} \mid A_{i j} \in \boldsymbol{A}_{i j}^{I}\right.$ for $\left.i=1, \ldots, m ; j=1, \ldots n\right\}$

An interval matrix is one that contains all real matrices, whose elements are obtained from all possible values between the lower and upper bound of its interval elements.

The mid-matrix and uncertainty of an interval matrix $\boldsymbol{A}^{\boldsymbol{I}}$ are defined as

$$
A^{c}=\frac{1}{2}(\underline{A}+\bar{A})
$$

And

$$
\Delta A=\frac{1}{2}(\bar{A}-\underline{A})
$$

Respectively. So an interval matrix can be expressed as

$$
\boldsymbol{A}^{\boldsymbol{I}}=A^{c}+\Delta \boldsymbol{A}^{\boldsymbol{I}}=\left[A^{c}-\Delta A, A^{c}+\Delta A\right]
$$

Where $\Delta \boldsymbol{A}^{I}=[-\Delta A, \Delta A]$.

Interval arithmetic operations on real numbers can be explicitly expressed as follows,

$$
\begin{aligned}
& \boldsymbol{x}^{\boldsymbol{I}}+\boldsymbol{y}^{\boldsymbol{I}}=[\underline{x}+\underline{y}, \bar{x}+\bar{y}], \\
& \boldsymbol{x}^{\boldsymbol{I}}-\boldsymbol{y}^{\boldsymbol{I}}=[\underline{x}-\bar{y}, \bar{x}-\underline{y}], \\
& \boldsymbol{x}^{I} \times \boldsymbol{y}^{I}=[\min \{\underline{x} \underline{y}, \bar{x} \bar{y}, \underline{x} \bar{y}, \bar{x} \underline{y}\}, \max \{\underline{x} \underline{y}, \bar{x} \bar{y}, \underline{x} \bar{y}, \bar{x} \underline{y}\}], \\
& \left(x^{I}\right)^{-1}=\left[\bar{x}^{-1}, \underline{x}^{-1}\right] \text { if } \underline{x}>0 \text { or } \bar{x}<0, \\
& x^{I} \div y^{I}=x^{I} \times\left(y^{I}\right)^{-1},
\end{aligned}
$$

For the elementary interval operations, division by an interval containing zero is not defined. Note that any real number $r$ is denoted by a degenerate interval $[r, r]$.

\section{StRUCTURAL DyNAMic RESPONSE ANALYSIS WITH INTERVAL PARAMETERS}

For a system under external loading, the general interval dynamic equation (Meirovoth 1980[7]; Weaver and Johnston 1987[11]) with $n$ degrees of freedom can be expressed as follows,

$$
\left[M^{I}\right] \ddot{X}^{I}(t)+\left[C^{I}\right] \dot{X}^{I}(t)+\left[K^{I}\right] X^{I}(t)=P^{I}(t),
$$

Where $\left[\boldsymbol{M}^{\boldsymbol{I}}\right]=\left(\boldsymbol{m}_{\boldsymbol{i j}}^{\boldsymbol{I}}\right),\left[\boldsymbol{C}^{\boldsymbol{I}}\right]=\left(\boldsymbol{c}_{\boldsymbol{i j}}^{\boldsymbol{I}}\right)$ and $\left[\boldsymbol{K}^{\boldsymbol{I}}\right]=\left(\boldsymbol{k}_{\boldsymbol{i j}}^{\boldsymbol{I}}\right)$ are the interval mass matrix, damping matrix and stiffness matrix; $P^{I}(t)=\left(p_{i}^{I}(t)\right)$ is the external load vector; $\boldsymbol{X}^{I}(\boldsymbol{t})=\left(\boldsymbol{x}_{\boldsymbol{i}}^{I}(\boldsymbol{t})\right)$, $\dot{X}^{I}(t)=\left(\dot{x}_{i}^{I}(t)\right)$ and $\ddot{X}^{I}(t)=\left(\ddot{x}_{i}^{I}(t)\right)$ are the displacement, velocity and acceleration vectors of the finite element assemblage, respectively. The damping matrix $\left[\boldsymbol{C}^{\boldsymbol{I}}\right]$ can be written as,

$$
\left[\boldsymbol{C}^{\boldsymbol{I}}\right]=a\left[\boldsymbol{K}^{\boldsymbol{I}}\right]+b\left[\boldsymbol{M}^{\boldsymbol{I}}\right],
$$

Where $a$ and $b$ are the coefficients of Rayleigh Damping.

The Laplace transform is used in this study to obtain the solution of Eq. (16). The Laplace transform is defined as follows,

$$
F(s)=\mathcal{L}\{f(t)\}=\int_{0}^{+\infty} e^{-s t} f(t) d t
$$

Where the parameter $s$ is a complex number, $s=\gamma+i \omega$, with real numbers $\gamma$ and $\omega$. And the inverse Laplace transform is defined as follows,

$$
f(t)=\mathcal{L}^{-1}\{F(s)\}=\frac{1}{2 \pi i} \int_{\gamma-i \omega}^{\gamma+i \omega} e^{s t} F(s) d s
$$

Where $\gamma$ is a real number so that the contour path of integration is in the region of convergence of $F(s)$ normally requiring $\gamma>\operatorname{Re}\left(S_{p}\right)$ for every singularity $S_{p}$ of $F(s)$ and $i^{2}=-1$. When using Laplace transform for Eq. (16), the Laplace transform of $\ddot{\boldsymbol{X}}^{I}(\boldsymbol{t})$ and $\dot{\boldsymbol{X}}^{I}(\boldsymbol{t})$ can be expressed as,

$$
\mathcal{L}\left\{\ddot{\boldsymbol{X}}^{I}(\boldsymbol{t})\right\}=s^{2} \mathcal{L}^{I}(s)-s \boldsymbol{X}^{I}(0)-\dot{\boldsymbol{X}}^{I}(0)
$$

And

$$
\mathcal{L}\left\{\dot{X}^{I}(t)\right\}=s \mathcal{L}^{I}(\mathrm{~s})-\dot{X}^{I}(0)
$$

After using Laplace transform, Eq. (16) becomes, $\quad\left(\left[\boldsymbol{M}^{I}\right] s^{2}+\left[\boldsymbol{C}^{I}\right] s+\left[\boldsymbol{K}^{I}\right]\right) \mathcal{L}^{I}(\boldsymbol{s})=\boldsymbol{F}^{I}(\boldsymbol{s})+$ $\left(\left[\boldsymbol{M}^{I}\right] s+\left[\boldsymbol{C}^{I}\right]\right) \boldsymbol{X}^{I}(0)+\left[\boldsymbol{M}^{I}\right] \dot{\boldsymbol{X}}^{I}(0)$

Where $\boldsymbol{L}^{\boldsymbol{I}}(\boldsymbol{s}), \boldsymbol{F}^{\boldsymbol{I}}(\boldsymbol{s}), \boldsymbol{X}^{\boldsymbol{I}}(0)$ and $\dot{\boldsymbol{X}}^{\boldsymbol{I}}(0)$ are the Laplace transform of $\boldsymbol{X}^{\boldsymbol{I}}(\mathrm{t})$, the Laplace transform of $\boldsymbol{P}^{I}(\boldsymbol{t})$, the system initial displacement and the system initial velocity, respectively. If all the initial conditions of the structure are zero, that means $\boldsymbol{X}^{I}(0)=0, \dot{\boldsymbol{X}}^{I}(0)=0$ and $\ddot{\boldsymbol{X}}^{I}(0)=0$, Eq. (22) can be written as,

$$
\left(\left[\boldsymbol{M}^{\boldsymbol{I}}\right] s^{2}+\left[\boldsymbol{C}^{\boldsymbol{I}}\right] s+\left[\boldsymbol{K}^{\boldsymbol{I}}\right]\right) \mathcal{L}^{\boldsymbol{I}}(\boldsymbol{s})=\boldsymbol{F}^{\boldsymbol{I}}(\boldsymbol{s})
$$

Using the Rayleigh Damping Eq. (17), Eq. (23) becomes, 


$$
\left(\left[\boldsymbol{M}^{\boldsymbol{I}}\right]\left(s^{2}+b s\right)+\left[\boldsymbol{K}^{\boldsymbol{I}}\right](a s+1)\right) \mathcal{L}^{I}(\boldsymbol{s})=\boldsymbol{F}^{\boldsymbol{I}}(\boldsymbol{s})
$$

The interval matrix $\mathcal{L}^{\boldsymbol{I}},\left[\boldsymbol{M}^{\boldsymbol{I}}\right],\left[\boldsymbol{K}^{\boldsymbol{I}}\right]$ and $\boldsymbol{F}^{\boldsymbol{I}}$ are expressed with mid-matrix and uncertainty, which are,

$$
\begin{gathered}
\mathcal{L}^{I}=\mathcal{L}^{c}+\Delta \mathcal{L}^{I} \\
{\left[\boldsymbol{M}^{I}\right]=M^{c}+\Delta \boldsymbol{M}^{I}} \\
{\left[\boldsymbol{K}^{I}\right]=K^{c}+\Delta \boldsymbol{K}^{I}} \\
\boldsymbol{F}^{I}=F^{c}+\Delta \boldsymbol{F}^{I}
\end{gathered}
$$

Substituting Eqs. (25), (26), (27) and (28) into Eq. (24), it leads to,

$$
\begin{gathered}
{\left[M^{c}\left(s^{2}+b s\right)+K^{c}(a s+1)\right] \mathcal{L}^{c}(s)} \\
+\left[\Delta \boldsymbol{M}^{I}\left(s^{2}+b s\right)+\Delta \boldsymbol{K}^{I}(a s+1)\right] \mathcal{L}^{c}(s) \\
+\left[M^{c}\left(s^{2}+b s\right)+K^{c}(a s+1)\right] \Delta \mathcal{L}^{I}(s) \\
+\left[\Delta \boldsymbol{M}^{I}\left(s^{2}+b s\right)+\Delta \boldsymbol{K}^{I}(a s+1)\right] \Delta \mathcal{L}^{I}(s)=F^{c}(s)+ \\
\Delta \boldsymbol{F}^{I}(s)
\end{gathered}
$$

It can be found that,

$$
\left[M^{c}\left(s^{2}+b s\right)+K^{c}(a s+1)\right] \mathcal{L}^{c}(s)=F^{c}(s)
$$

If the second order of uncertain terms in Eq. (29) are neglected, and Eq. (30) is considered, Eq. (29) can be written as,

$$
\begin{aligned}
A \times \Delta \mathcal{L}^{I}(\boldsymbol{s}) & =\Delta \boldsymbol{F}^{I}(s)-\left[\Delta \boldsymbol{M}^{I}\left(s^{2}+b s\right)+\Delta \boldsymbol{K}^{I}(a s+\right. \\
1)] \mathcal{L}^{c}(s) & \\
A & =M^{c}\left(s^{2}+b s\right)+K^{c}(a s+1)
\end{aligned}
$$

Therefore, from Eq. (31), the uncertainty of $\boldsymbol{L}^{I}(\boldsymbol{s})$ can be obtained,

$$
\begin{aligned}
& \Delta \mathcal{L}^{I}(\boldsymbol{s})=A^{-1} \Delta \boldsymbol{F}^{I}(s)-A^{-1} \Delta \boldsymbol{M}^{I}\left(s^{2}+b s\right) \mathcal{L}^{c}(s)- \\
& A^{-1} \Delta \boldsymbol{K}^{I}(a s+1) \mathcal{L}^{c}(s)
\end{aligned}
$$

After taking the inverse Laplace transform of each term of Eq. (33), the dynamic response of the structure can be obtained,

$$
\begin{aligned}
& \Delta \boldsymbol{X}^{\boldsymbol{I}}(\boldsymbol{t})=\mathcal{L}^{-1}\left\{A^{-1} \Delta \boldsymbol{F}^{\boldsymbol{I}}(s)\right\} \\
&-\mathcal{L}^{-1}\left\{A^{-1} \Delta \boldsymbol{M}^{I}\left(s^{2}+b s\right) \mathcal{L}^{c}(s)\right\}-\mathcal{L}^{-1}\left\{A^{-1} \Delta \boldsymbol{K}^{I}(a s+\right. \\
&\left.1) \mathcal{L}^{c}(s)\right\}
\end{aligned}
$$

It should be noted that it is difficult to integrate the terms with uncertainty. Thus, a special treatment of matrix is used to extract the uncertainties outside the integration, for example the term $A^{-1} \Delta \boldsymbol{M}^{I}\left(s^{2}+b s\right) \mathcal{L}^{c}(s)$ which can be shown as below,

$$
\begin{aligned}
\mathcal{L}^{-1}\left\{A^{-1} \Delta \boldsymbol{M}^{\boldsymbol{I}}\left(s^{2}+b s\right) \mathcal{L}^{c}(s)\right\} \\
=\mathcal{L}^{-1}\left\{A^{-1}\left(s^{2}+b s\right) M^{c} \boldsymbol{\delta}^{I} \mathcal{L}^{c}(s)\right\} \\
=\mathcal{L}^{-1}\left\{A^{-1}\left(s^{2}+b s\right) M^{c} \mathcal{L}^{c \prime}(s)\right\} \boldsymbol{\delta}^{\prime \prime}
\end{aligned}
$$

Where $\mathcal{L}^{c^{\prime}}(s)$ and $\boldsymbol{\delta}^{\boldsymbol{I}}$ are the new matrix for substituting the term $\boldsymbol{\delta}^{I} \mathcal{L}^{c}(s)$, and the matrix dimension of $\mathcal{L}^{c^{\prime}}(s)$ and $\boldsymbol{\delta}^{\boldsymbol{I}}$ are same as $\boldsymbol{\delta}^{\boldsymbol{I}}$ and $\mathcal{L}^{c}(s)$, respectively.

\section{NUMERICAL SIMULATIONS}

In order to illustrate the effectiveness of the presented interval method for the dynamic responses of the structures with uncertainty, a three-story frame tower structure without damping which is shown in Figure 1 is analyzed. Each story has the same mass $M_{1}=M_{2}=M_{3}=100 \mathrm{~kg}$ with $5 \%$ uncertainty. The system stiffness is $K_{1}=K_{2}=K_{3}=$ $1000 \mathrm{KN} / \mathrm{m}$ with $10 \%$ uncertainty. The stiffnessand the mass density of each element are assumed to vary independently.It is assumed that there is a harmonic external loading $F=\cos (3 t)$ acting on the first story in horizontal direction with the initial conditions $\boldsymbol{x}^{I}(0)=0, \dot{\boldsymbol{x}}^{I}(0)=0$. The displacement response of the first story in the horizontal direction is calculated and shown in Figure 2 and Figure 3.

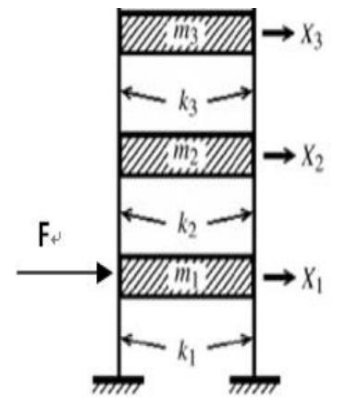

FIGURE I. THREE-STORY FRAME TOWER.

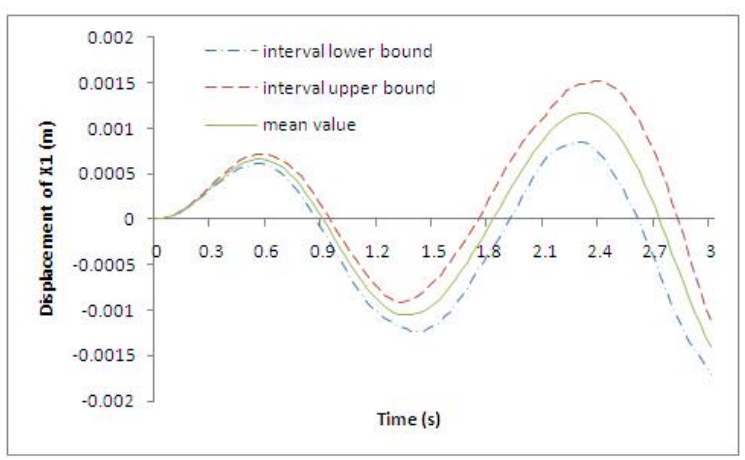

FIGURE II. RESPONSE OF FIRST STORY WITHOUT DAMPING IN HORIZONTAL DIRECTION IN 3 SECONDS.

The response range of the first story in the horizontal direction in the first 3 seconds is shown in Figure 2. In the first 3 seconds that is the region before divergent response, the variations of the upper and lower bounds of the dynamic response are small. That is to say, the presented method has a good robustness with respect to interval parameters. Figure 3 describes the response range of the first story in the horizontal direction in the first 10 seconds. It can be found that the dynamic responses of the undamped structure are divergent due to interval parameters. This divergent phenomenon is caused by the free vibration of the structure. 


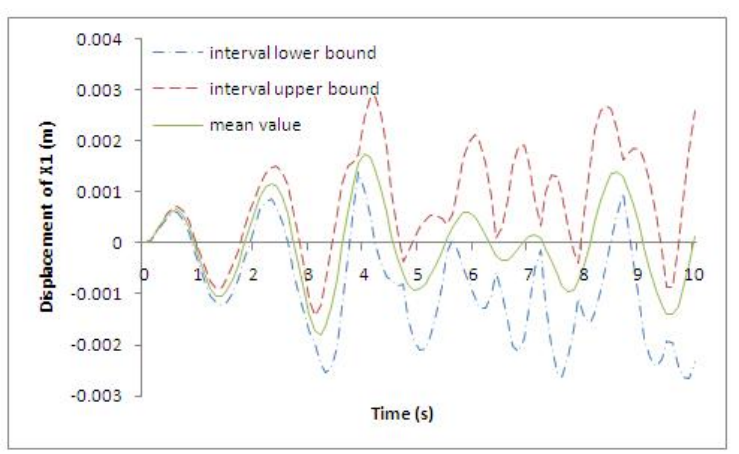

FIGURE III. RESPONSE OF FIRST STORY WITHOUT DAMPING IN HORIZONTAL DIRECTION IN 10 SECONDS.

Consider the same structure shown in Figure 1. The damping matrix is assumed to be $\left[\boldsymbol{C}^{\boldsymbol{I}}\right]=0.01 \times\left[\boldsymbol{K}^{\boldsymbol{I}}\right]+1 \times$ $\left[\boldsymbol{M}^{\boldsymbol{I}}\right]$. The displacement response of the first story in the horizontal direction is shown in Figure 4.

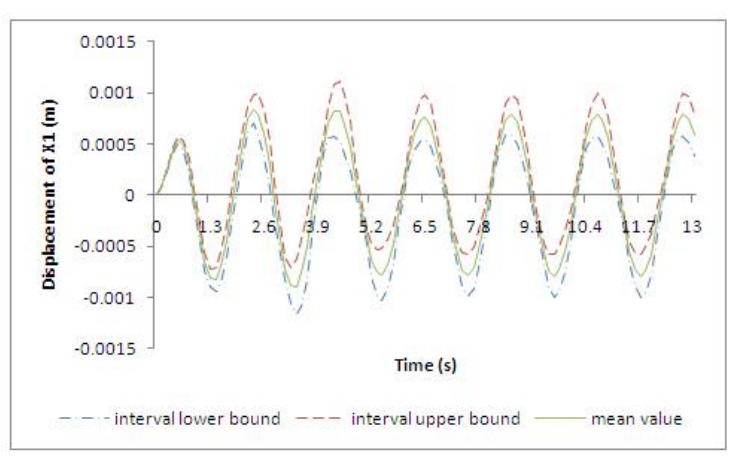

FIGURE IV. RESPONSE OF FIRST STORY WITH DAMPING IN HORIZONTAL DIRECTION IN 10 SECONDS

In Figure 4, the vertical axis is the displacement of the first story of the structure in the horizontal direction and the horizontal axis is the time from 0 to 13 seconds. It can be observed that the dynamic responses of the structure are convergent under damping. This is different from the undamped system. The reason of this phenomenon is that the responses of damping system are controlled by the external force. Comparing the variations of the upper and lower bounds, the presented method holds the high computing accuracy in interval calculation.

\section{CONCLUSION}

A new interval method has been presented to determine the range of vibration responses of structures with interval parameters. The effectiveness of the proposed method has been demonstrated by a numerical example of a three-story structure. The results have shown that the range between upper and lower bonds of dynamic responses due to uncertain system parameters is narrow and acceptable. Since the presented method neglects the higher order terms in the expansion of functions, the application of the approach is limited to the cases where the uncertainties of the interval parameters are small $(<10 \%)$.

\section{REFERENCES}

[1] Au, S. K. (2004), "Reliability-based design sensitivity by efficient simulation”. Computers and Structures.83 1048-1061.

[2] Alefeld, G. and Herzberger, J. (1983), Introduction to Interval Computations, York: Academic Press).

[3] Chen, S. H., Lian, H. D. and Yang, X. W. (2002), "Dynamic response analysis for structures with interval parameters”.StructEngMech, 133 299-312.

[4] Chen, S. H. and Wu, J. (2004), "Interval optimization of dynamic response for structures with interval parameters”, Computer and Structures,82 1-11.

[5] Chen, S. H. and Zhang, X. M. (2006), "Dynamic response of closedloop system with uncertain parameters using interval finite element method”, Journal of Engineering Mechanics,132(8), pp. 830-840.

[6] Hansen, E. (1992), Global Optimization Using Interval Analysis, (New York: Marcel Dekker).

[7] Meirovotch, L. (1980), Computational Methods in Structural Dynamics, (Sijthoff\&Noordhoff).

[8] Moore, R. E. (1966), Interval Analysis, (New York: Prentice-Hall, Englewood Cliffs).

[9] Qiu, Z. P. and Wang, X. J. (2003), “Comparison of dynamic response of structures with uncertain-but-bounded parameters using nonprobabilistic interval analysis method and probabilistic approach”, International Journal of Solids and Structures,40 5423-5439.

[10] Qiu, Z. P. and Wang, X. J. (2005), "Parameter perturbation method for dynamic responses of structures with uncertain-but-bounded parameters based on interval analysis”, International Journal of Solids and Structures, 42 4958-4970.

[11] Weaver, Jr. W. and Johnston, P. R. (1987) Structural Dynamics by Finite Elements, (New Jersey: Prentice-Hall Inc.). 